\title{
Corrigendum: External
} Human-Machine Interfaces for Autonomous Vehicle-to-Pedestrian Communication: A Review of Empirical Work

\author{
Alexandros Rouchitsas * and Håkan Alm \\ Humans and Technology Division, Luleå University of Technology, Luleå, Sweden \\ Keywords: traffic interaction, human-vehicle interaction, autonomous vehicles, vehicle-to-pedestrian \\ communication, external human-machine interfaces, vulnerable road users
}

\section{A Corrigendum on}

External Human-Machine Interfaces for Autonomous Vehicle-to-Pedestrian Communication: A Review of Empirical Work

by Rouchitsas, A., and Alm, H. (2019). Front. Psychol. 10:2757. doi: 10.3389/fpsyg.2019.02757

\section{OPEN ACCESS}

Edited and reviewed by: Anton Nijholt,

University of Twente, Netherlands

${ }^{*}$ Correspondence:

Alexandros Rouchitsas alexandros.rouchitsas@ltu.se

Specialty section:

This article was submitted to

Human-Media Interaction,

a section of the journal

Frontiers in Psychology

Received: 22 June 2020

Accepted: 27 August 2020

Published: 16 October 2020

Citation:

Rouchitsas A and Alm H (2020)

Corrigendum: External

Human-Machine Interfaces for Autonomous Vehicle-to-Pedestrian

Communication: A Review of Empirical Work. Front. Psychol. 11:575151. doi: 10.3389/fpsyg.2020.575151
In the original article, there were five errors.

1. The word "only" was used instead of "mainly."

A correction has been made to section External Human-Machine Interfaces Evaluated Via Empirical Studies, sub-section Studies Employing Physical Prototypes. The corrected sentence reads as follows:

"While the aforementioned studies have used mainly subjective measures to assess interface effectiveness, Clamann et al. (2017) evaluated a communication interface by using an objective measure, namely decision time, alongside ratings and interviews."

2. The word "reaction" was used instead of "decision".

A correction has been made to External Human-Machine Interfaces Evaluated Via Empirical Studies, sub-section VR-Based Studies. The corrected sentence reads as follows:

"All designs proved to be efficient, as evidenced by shorter decision times when compared to the baseline condition (autonomous vehicle without interface)."

3. The word "experimental" was used instead of "behavioral".

A correction has been made to Discussion section. The corrected sentence reads as follows:

"Interestingly, the most convincing evidence were obtained largely from studies conducted in laboratory settings, namely monitor-based and VR-based studies, that utilized mainly objective measures, like reaction time, duration, and accuracy, in the context of behavioral tasks."

Additionally, there was an error in Table 1 as published. The second-to-final version of Table 1 was included in the original article. The final version of the table appears below.

The authors apologize for these errors and state that they do not change the scientific conclusions of the article in any way. The original article has been updated. 


\section{REFERENCES}

Ackermann, C., Beggiato, M., Schubert, S., and Krems, J. F. (2019). An experimental study to investigate design and assessment criteria: what is important for communication between pedestrians and automated vehicles? Appl. Ergon. 75, 272-282. doi: 10.1016/j.apergo.2018. 11.002

Böckle, M. P., Brenden, A. P., Klingegård, M., Habibovic, A., and Bout, M. (2017). "SAV2P: exploring the impact of an interface for shared automated vehicles on pedestrians' experience," in Proceedings of the 9th International Conference on Automotive User Interfaces and Interactive Vehicular Applications Adjunct (New York, NY: ACM), 136-140.

Chang, C. M., Toda, K., Igarashi, T., Miyata, M., and Kobayashi, Y. (2018). "A video-based study comparing communication modalities between an autonomous car and a pedestrian," in Adjunct Proceedings of the 10th International Conference on Automotive User Interfaces and Interactive Vehicular Applications (New York, NY: ACM), 104-109.

Chang, C. M., Toda, K., Sakamoto, D., and Igarashi, T. (2017). "Eyes on a car: an interface design for communication between an autonomous car and a pedestrian," in Proceedings of the 9th International Conference on Automotive User Interfaces and Interactive Vehicular Applications (New York, NY: ACM), 65-73.

Charisi, V., Habibovic, A., Andersson, J., Li, J., and Evers, V. (2017). "Children's views on identification and intention communication of self-driving vehicles," in Proceedings of the 2017 Conference on Interaction Design and Children (New York, NY: ACM), 399-404.

Clamann, M., Aubert, M., and Cummings, M. L. (2017). "Evaluation of vehicle-topedestrian communication displays for autonomous vehicles," in Proceedings of the 96th Annual Transportation Research Board Meeting (Washington, DC).

Costa, G. (2017). Designing Framework for Human-Autonomous Vehicle Interaction. Master's thesis, Designing Framework for Human-Autonomous Vehicle Interaction, Minato.

de Clercq, K., Dietrich, A., Núñez Velasco, J. P., de Winter, J., and Happee, R. (2019). External human-machine interfaces on automated vehicles: effects on pedestrian crossing decisions. Hum. Factors 61, 1353-1370. doi: $10.1177 / 0018720819836343$

Deb, S., Strawderman, L. J., and Carruth, D. W. (2018). Investigating pedestrian suggestions for external features on fully autonomous vehicles: a virtual reality experiment. Transp. Res. Part F Traffic Psychol. Behav. 59, 135-149. doi: 10.1016/j.trf.2018.08.016

Fridman, L., Mehler, B., Xia, L., Yang, Y., Facusse, L. Y., and Reimer, B. (2017). To walk or not to walk: crowdsourced assessment of external vehicle-to-pedestrian displays. arXiv [Preprint].

Habibovic, A. (2018). Communicating intent of automated vehicles to pedestrians. Front. Psychol. 9:1336. doi: 10.3389/fpsyg.2018.01336
Hensch, A. C., Neumann, I., Beggiato, M., Halama, J., and Krems, J. F. (2019) "How should automated vehicles communicate? effects of a light-based communication approach in a wizard-of-oz study," in Proceedings of the International Conference on Applied Human Factors and Ergonomics. (Cham: Springer), 79-91. doi: 10.1007/978-3-030-20503-4_8

Hudson, C. R., Deb, S., Carruth, D. W., McGinley, J., and Frey, D. (2018). "Pedestrian perception of autonomous vehicles with external interacting features," In Proceedings of the International Conference on Applied Human Factors and Ergonomics. (Cham: Springer), 33-39. doi: 10.1007/978-3-319-94334-3_5

Li, Y., Dikmen, M., Hussein, T. G., Wang, Y., and Burns, C. (2018). “To cross or not to cross: urgency-based external warning displays on autonomous vehicles to improve pedestrian crossing safety," in Proceedings of the 10th International Conference on Automotive User Interfaces and Interactive Vehicular Applications (New York, NY: ACM), 188-197.

Mahadevan, K., Somanath, S., and Sharlin, E. (2018). "Communicating awareness and intent in autonomous vehicle-pedestrian interaction," in Proceedings of the 2018 CHI Conference on Human Factors in Computing Systems (New York, NY: ACM), 429.

Othersen, I., Conti-Kufner, A., Dietrich, A., Maruhn, P., and Bengler, K. (2018). "Designing for automated vehicle and pedestrian communication," in Proceedings of the Perspectives on eHMIs from Older and Younger Persons (Netherlands: HFES Europe Annual Meeting).

Petzoldt, T., Schleinitz, K., and Banse, R. (2018). Potential safety effects of a frontal brake light for motor vehicles. IEEE Intell. Trans. Sys. 12, 449-453. doi: 10.1049/iet-its.2017.0321

Song, Y. E., Lehsing, C., Fuest, T., and Bengler, K. (2018). "External HMIs and their Effect on the Interaction Between Pedestrians and Automated Vehicles," in Proceedings of the International Conference on Intelligent Human Systems Integration (Cham: Springer), 13-18. doi: 10.1007/978-3-319-73888-8_3

Stadler, S., Cornet, H., Theoto, T. N., and Frenkler, F. (2019). "A tool, not a toy: using virtual reality to evaluate the communication between autonomous vehicles and pedestrians," in Augmented Reality and Virtual Reality, eds M. tom Dieck, T. Jung (Cham: Springer), 203-216. doi: 10.1007/978-3-030-06246-0_15

Zhang, J., Vinkhuyzen, E., and Cefkin, M. (2017). "Evaluation of an autonomous vehicle external communication system concept: a survey study," in Proceedings of the International Conference on Applied Human Factors and Ergonomics. (Cham: Springer), 650-661. doi: 10.1007/978-3-319-60441-1_63

Copyright (c) 2020 Rouchitsas and Alm. This is an open-access article distributed under the terms of the Creative Commons Attribution License (CC BY). The use, distribution or reproduction in other forums is permitted, provided the original author(s) and the copyright owner(s) are credited and that the original publication in this journal is cited, in accordance with accepted academic practice. No use, distribution or reproduction is permitted which does not comply with these terms. 


\begin{tabular}{|c|c|c|c|c|c|c|c|c|c|c|c|c|c|c|}
\hline \multirow[t]{2}{*}{ Studies } & \multicolumn{3}{|c|}{ Stimulus delivery } & \multicolumn{5}{|c|}{ Interface parameters } & \multicolumn{4}{|c|}{ Evaluation procedures } & \multicolumn{2}{|c|}{ Measures } \\
\hline & $\begin{array}{l}\text { Physical } \\
\text { Prototype }\end{array}$ & $\begin{array}{l}\text { Monitor- } \\
\text { based }\end{array}$ & $\begin{array}{l}\text { VR- } \\
\text { based }\end{array}$ & Technology & Location & Content type & Information type & Message coding & Modality & Behavioral task & Online survey & Questionnaire & Objective & Subjective \\
\hline $\begin{array}{l}\text { Hensch et al. } \\
\text { (2019) }\end{array}$ & $\checkmark$ & & & Display & Roof & Information & Mode, intention & Lights & Visual & $\begin{array}{l}\text { Intention } \\
\text { identification }\end{array}$ & & $\begin{array}{l}\text { Comprehensibility, } \\
\text { trust, safety, } \\
\text { usefulness }\end{array}$ & & $\begin{array}{l}\text { Likert scales, } \\
\text { interview }\end{array}$ \\
\hline Costa (2017) & $\checkmark$ & & & $\begin{array}{l}\text { Cardboard, } \\
\text { speaker }\end{array}$ & Hood, bumper & Advice & & $\begin{array}{l}\text { Textual, pictorial, } \\
\text { sounds }\end{array}$ & $\begin{array}{l}\text { Visual, } \\
\text { auditory }\end{array}$ & Street-crossing & & & Frequency & \\
\hline $\begin{array}{l}\text { Mahadevan et al. } \\
\text { (2018) }\end{array}$ & $\checkmark$ & & & $\begin{array}{l}\text { Light strip, } \\
\text { display, LEDs, } \\
\text { printed hand, } \\
\text { mobile phone, } \\
\text { speaker }\end{array}$ & $\begin{array}{l}\text { Windshield, } \\
\text { hood, roof, } \\
\text { street surface, } \\
\text { pedestrian's } \\
\text { mobile phone }\end{array}$ & Information & $\begin{array}{l}\text { Pedestrian } \\
\text { acknowledgment, } \\
\text { intention }\end{array}$ & $\begin{array}{l}\text { Lights, speech, } \\
\text { vibration, } \\
\text { gesture, pictorial }\end{array}$ & $\begin{array}{l}\text { Visual, } \\
\text { auditory, } \\
\text { haptic }\end{array}$ & $\begin{array}{l}\text { Crossing } \\
\text { intention }\end{array}$ & & $\begin{array}{l}\text { Effectiveness, } \\
\text { confidence }\end{array}$ & & $\begin{array}{l}\text { Likert scales, } \\
\text { interview }\end{array}$ \\
\hline Habibovic (2018) & $\checkmark$ & & & Light strip & Windshield & Information & Mode, intention & Lights & Visual & Street-crossing & & Safety & & $\begin{array}{l}\text { Likert scales, } \\
\text { interview }\end{array}$ \\
\hline $\begin{array}{l}\text { Clamann et al. } \\
\text { (2017) }\end{array}$ & $\checkmark$ & & & Display & Radiator grille & $\begin{array}{l}\text { Information, } \\
\text { advice }\end{array}$ & Speed & Textual, pictorial & Visual & Street-crossing & & Effectiveness & $\begin{array}{l}\text { Decision } \\
\text { time }\end{array}$ & Interview \\
\hline Li et al. (2018) & & $\checkmark$ & & Display & $\begin{array}{l}\text { Windshield, } \\
\text { radiator grille, } \\
\text { vehicle sides }\end{array}$ & Advice & & Lights & Visual & & $\begin{array}{l}\text { Situational urgency, } \\
\text { crossing intention }\end{array}$ & & & $\begin{array}{l}\text { Numeric scales, } \\
\text { interview }\end{array}$ \\
\hline Zhang et al. (2017) & & $\checkmark$ & & Light strip & $\begin{array}{l}\text { Front doors, } \\
\text { hood }\end{array}$ & Information & Intention & Lights & Visual & & $\begin{array}{l}\text { Intention identification, } \\
\text { effectiveness }\end{array}$ & & & Interview \\
\hline Song et al. (2018) & & $\checkmark$ & & Display & Radiator grille & Advice & & Textual, pictorial & Visual & & $\begin{array}{l}\text { Crossing intention, } \\
\text { preference }\end{array}$ & & $\begin{array}{l}\text { Reaction } \\
\text { time, } \\
\text { frequency }\end{array}$ & Interview \\
\hline $\begin{array}{l}\text { Fridman et al. } \\
\text { (2017) }\end{array}$ & & $\checkmark$ & & $\begin{array}{l}\text { Light strip, } \\
\text { display, } \\
\text { projection, } \\
\text { vehicle lights and } \\
\text { signals }\end{array}$ & $\begin{array}{l}\text { Windshield, } \\
\text { headlights, fog } \\
\text { lights, directional } \\
\text { signals, radiator } \\
\text { grille, bumper, } \\
\text { street surface }\end{array}$ & $\begin{array}{l}\text { Information } \\
\text { advice }\end{array}$ & Intention & $\begin{array}{l}\text { Textual, pictorial, } \\
\text { lights }\end{array}$ & Visual & & Crossing intention & & $\begin{array}{l}\text { Error rates, } \\
\text { reaction } \\
\text { time }\end{array}$ & \\
\hline $\begin{array}{l}\text { Ackermann et al. } \\
\text { (2019) }\end{array}$ & & $\checkmark$ & & $\begin{array}{l}\text { Light strip, } \\
\text { display, } \\
\text { projection }\end{array}$ & $\begin{array}{l}\text { Windshield, } \\
\text { radiator grille, } \\
\text { street surface }\end{array}$ & $\begin{array}{l}\text { Information, } \\
\text { advice }\end{array}$ & Mode & $\begin{array}{l}\text { Lights, textual, } \\
\text { pictorial }\end{array}$ & Visual & & & $\begin{array}{l}\text { Comprehensibility, } \\
\text { recognizability, } \\
\text { ambiguousness, } \\
\text { comfort }\end{array}$ & & $\begin{array}{l}\text { Numeric scales, } \\
\text { interview }\end{array}$ \\
\hline $\begin{array}{l}\text { Petzoldt et al. } \\
\text { (2018) }\end{array}$ & & $\checkmark$ & & Light strip & $\begin{array}{l}\text { Above license } \\
\text { plate }\end{array}$ & Information & Deceleration & Lights & Visual & $\begin{array}{l}\text { Deceleration } \\
\text { detection }\end{array}$ & & Usefulness, safety & $\begin{array}{l}\text { Error rates, } \\
\text { reaction } \\
\text { time }\end{array}$ & Likert scales \\
\hline Chang et al. (2018) & & $\checkmark$ & & $\begin{array}{l}\text { Light strip, } \\
\text { display, } \\
\text { projection, } \\
\text { rotating vehicle } \\
\text { lights }\end{array}$ & $\begin{array}{l}\text { Windshield, } \\
\text { radiator grille, } \\
\text { street surface, } \\
\text { headlights }\end{array}$ & Information & Intention & $\begin{array}{l}\text { Lights, textual, } \\
\text { pictorial, } \\
\text { anthropomorphism }\end{array}$ & Visual & $\begin{array}{l}\text { Intention } \\
\text { identification }\end{array}$ & & Intelligibility & Error rates & Likert scales \\
\hline Charisi et al. (2017) & & $\checkmark$ & & $\begin{array}{l}\text { Display, light } \\
\text { strip, projection, } \\
\text { vehicle lights and } \\
\text { signals }\end{array}$ & $\begin{array}{l}\text { Windshield, } \\
\text { headlights, } \\
\text { directional } \\
\text { signals, street } \\
\text { surface }\end{array}$ & Information & Intention & $\begin{array}{l}\text { Lights, textual, } \\
\text { pictorial, } \\
\text { anthropomorphism }\end{array}$ & Visual & $\begin{array}{l}\text { Intention } \\
\text { identification }\end{array}$ & & $\begin{array}{l}\text { Intention } \\
\text { identification }\end{array}$ & Error rates & Interview \\
\hline
\end{tabular}




\begin{tabular}{|c|c|c|c|c|c|c|c|c|c|c|c|c|c|c|}
\hline \multirow[t]{2}{*}{ Studies } & \multicolumn{3}{|c|}{ Stimulus delivery } & \multicolumn{5}{|c|}{ Interface parameters } & \multicolumn{4}{|c|}{ Evaluation procedures } & \multicolumn{2}{|c|}{ Measures } \\
\hline & $\begin{array}{l}\text { Physical } \\
\text { Prototype }\end{array}$ & $\begin{array}{l}\text { Monitor- } \\
\text { based }\end{array}$ & $\begin{array}{l}\text { VR- } \\
\text { based }\end{array}$ & Technology & Location & Content type & Information type & Message coding & Modality & Behavioral task & Online survey & Questionnaire & Objective & Subjective \\
\hline $\begin{array}{l}\text { de Clercq et al. } \\
\text { (2019) }\end{array}$ & & & $\checkmark$ & $\begin{array}{l}\text { Display, vehicle } \\
\text { lights and signals }\end{array}$ & $\begin{array}{l}\text { Radiator grille, } \\
\text { frontal brake } \\
\text { lights }\end{array}$ & $\begin{array}{l}\text { Information } \\
\text { advice }\end{array}$ & Intention & $\begin{array}{l}\text { Textual, lights, } \\
\text { pictorial }\end{array}$ & Visual & Safety-reporting & & Safety, preference & Duration & Interview \\
\hline $\begin{array}{l}\text { Hudson et al. } \\
\text { (2018) }\end{array}$ & & & $\checkmark$ & Display, speaker & Hood & Advice & & $\begin{array}{l}\text { Textual, pictorial, } \\
\text { speech, music }\end{array}$ & $\begin{array}{l}\text { Visual, } \\
\text { auditory }\end{array}$ & Street-crossing & & Preference & & Interview \\
\hline Deb et al. (2018) & & & $\checkmark$ & Display, speaker & Hood & $\begin{array}{l}\text { Information } \\
\text { advice }\end{array}$ & Intention & $\begin{array}{l}\text { Lights, pictorial, } \\
\text { speech, sounds, } \\
\text { music }\end{array}$ & $\begin{array}{l}\text { Visual, } \\
\text { auditory }\end{array}$ & Street-crossing & & $\begin{array}{l}\text { Safety, } \\
\text { acceptance }\end{array}$ & $\begin{array}{l}\text { Decision } \\
\text { time, } \\
\text { duration }\end{array}$ & $\begin{array}{l}\text { Likert scales, } \\
\text { interview }\end{array}$ \\
\hline $\begin{array}{l}\text { Stadler et al. } \\
\text { (2019) }\end{array}$ & & & 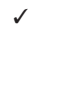 & Display & Radiator grille & Advice & & $\begin{array}{l}\text { Lights, textual, } \\
\text { pictorial, }\end{array}$ & Visual & Street-crossing & & Satisfaction & $\begin{array}{l}\text { Error rates, } \\
\text { decision } \\
\text { time }\end{array}$ & $\begin{array}{l}\text { Numeric scales, } \\
\text { interview }\end{array}$ \\
\hline $\begin{array}{l}\text { Othersen et al. } \\
\text { (2018) }\end{array}$ & & & $s$ & Display & Radiator grille & Information & $\begin{array}{l}\text { Pedestrian } \\
\text { detection, } \\
\text { intention }\end{array}$ & Lights, pictorial & Visual & Street-crossing & & $\begin{array}{l}\text { Effectiveness, } \\
\text { understandability, } \\
\text { perceptibility, } \\
\text { safety, appeal }\end{array}$ & $\begin{array}{l}\text { Decision } \\
\text { time }\end{array}$ & Interview \\
\hline $\begin{array}{l}\text { Chang et al. } \\
\text { (2017) }\end{array}$ & & & $s$ & $\begin{array}{l}\text { Rotating vehicle } \\
\text { lights }\end{array}$ & Headlights & Information & $\begin{array}{l}\text { Pedestrian } \\
\text { acknowledgment, } \\
\text { intention }\end{array}$ & Anthropomorphism & Visual & $\begin{array}{l}\text { Crossing } \\
\text { intention }\end{array}$ & & $\begin{array}{l}\text { Effectiveness, } \\
\text { safety }\end{array}$ & $\begin{array}{l}\text { Error rates, } \\
\text { reaction } \\
\text { time }\end{array}$ & $\begin{array}{l}\text { Likert scales, } \\
\text { interview }\end{array}$ \\
\hline $\begin{array}{l}\text { Böckle et al. } \\
\text { (2017) }\end{array}$ & & & $\checkmark$ & $\begin{array}{l}\text { Light strip, } \\
\text { speaker }\end{array}$ & Vehicle corners & Information & Intention & Lights, sounds & $\begin{array}{l}\text { Visual, } \\
\text { auditory }\end{array}$ & Street-crossing & & $\begin{array}{l}\text { Safety, comfort, } \\
\text { effectiveness }\end{array}$ & $\begin{array}{l}\text { Decision } \\
\text { time }\end{array}$ & $\begin{array}{l}\text { Likert scales, } \\
\text { interview }\end{array}$ \\
\hline
\end{tabular}

